BULL. AUSTRAL. MATH. SOC.

\title{
A generalization of Lagrange multipliers: Corrigendum
}

\section{B.D. Craven}

There is a lacuna in the proof of Lemma 1 of [1]; the projector $q$ is assumed without proof. An alternative, valid proof is as follows.

LEMMA 1. Let $S, U_{0}, V_{0}$ be real Banach spaces; let $A: S \rightarrow U_{0}$ and $B: S \rightarrow V_{0}$ be continuous linear maps, whose null spaces are $\mathrm{N}(A)$ respectively $\mathrm{N}(B)$; let $\mathrm{N}(A) \subset \mathrm{N}(B)$ : let $A$ map $S$ onto $U_{0}$. Then there exists a continuous linear map $C: U_{0} \rightarrow V_{0}$ such that $B=C \circ \mathrm{A}$.

Proof. Let $p$ denote the canonical projector of $S$ onto the quotient space $S / N(A)$; define $A_{0}: S / N(A) \rightarrow V$ by $A_{0}(x+N(A))=A x$; then $A=A_{0} \circ P$, and $A_{0}$ is a continuous bijection of $S / N(A)$ onto $U_{0}$. Hence $A_{0}^{-1}$ exists, continuous by Banach's bounded inverse theorem. Define $g: S / N(A) \rightarrow V_{0}$ by $g(x+N(A))=B x$, for each $x \in S$. This definition is unique since $B x=0$ for each $x \in N(A) \subset N(B)$; and then $g \circ p=B$, so $g$ is continuous since $B$ is continuous and $p$ is open. Define $C=g \circ A_{0}^{-1}: V \rightarrow W$. Then $C$ is continuous, and

$$
C \circ A=\left(g \circ A_{0}^{-1}\right) \circ\left(A_{0} \circ p\right)=g \circ p=B \text {. }
$$

Also the last line of page 354 of [1] should read: $\varphi^{\prime}(0)$ is invertible for sufficiently small $\|w\|$, since $A$ is invertible, and $\|\varphi(0)\|=\|h(0, w)\|<\varepsilon$ if ...

Received 16 November 1977. 


\title{
Reference
}

[1] B.D. Craven, "A generalization of Lagrange multipliers", BuZZ. Austral. Math. Soc. 3 (1970), 353-362.

\author{
Department of Mathematics, \\ University of Melbourne, \\ Parkville, \\ victoria.
}

